\title{
SIFAT-SIFAT DASAR MATRIKS SKEW HERMITIAN
}

Basic Properties of Skew Hermitian Matrices

\author{
LIDIA SALAKA ${ }^{1}$, HENRY W. M. PATTY ${ }^{2}$, MOZART WINSTON TALAKUA ${ }^{3}$ \\ ${ }^{1}$ Mahasiswa JurusanMatematika FMIPA UNPATTI \\ ${ }^{2,3}$ StafJurusan Matematika FMIPA UNPATTI \\ Jl. Ir. M. Putuhena, KampusUnpatti, Poka-Ambon, Maluku \\ E-mail: henrywmpatty81@gmail.com
}

\begin{abstract}
ABSTRAK
Matriks didefinisikan sebagai susunan persegi panjang dari elemen-elemen yang diatur dalam baris dan kolom. Matriks dengan elemen-elemen penyusunnya merupakan bilangan kompleks dikenal dengan matriks bilangan kompleks. Salah satu bentuk khusus dari matriks bilangan kompleks adalah matriks Skew Hermitian beserta sifat-sifatnya yang menjadikan matriks tersebut berbeda dengan matriks real. Penelitian ini membahas bagaimana mengetahui bentuk dari matriks Skew Hermitian, serta sifat-sifat aljabar matriks yang berlaku pada matriks Skew Hermitian, dengan tahapan penelitian sebagai berikut: mengubah matriks Hermitian menjadi matriks Skew Hermitian dengan cara mengenakan operasi pergandaan skalar $i$ (bilangan imajiner) pada matriks Hermitian, menyusun sifatsifat dasar matriks Skew Hermitian berdasarkan sifat dan definisi dari elemen-elemen penyusunnya. Hasil penelitian menunjukan bahwa sebuah matriks bujursangkar merupakan matriks Skew Hermitian jika setiap elemen-elemen penyusunnya merupakan bilangan kompleks beserta transpose konjugatnya dan matriks tersebut identik dengan negatif matriks transpose konjugatnya. Keterkaitannya dengan bentuk matriks lainnya juga merupakan suatu sifat yang berlaku pada matriks Skew Hermitian.
\end{abstract}

Kata kunci : bilangan kompleks, konjugat transpose, matriks, matriks Hermitian

\section{PENDAHULUAN}

Dalam perkembangan aljabar telah ditemukan beberapa bentuk matriks khusus dengan sifat-sifatnya yang dapat digunakan untuk meneliti perkembangan aljabar matriks. Salah satu diantaranya adalah bentuk khusus dari matriks bilangan kompleks beserta sifatsifatnya yang menjadikan matriks tersebut berbeda dengan matriks real. Salah satu bentuk dari matriks bilangan kompleks adalah matriks Hermitian yang ditemukan pada tahun 1855 oleh Charles Hermite, yang menyatakan bahwa suatu matriks Hermitian adalah suatu matriks kompleks berukuran $n \times n$ yang memiliki nilai yang sama dengan matriks transpose konjugatnya, dengan diagonal utamanya adalah bilangan real.

Seperti diketahui bahwa operasi matriks tidak terlepas dari operasi-operasi penjumlahan, operasi pergandaan, dan operasi pergandaan skalar. Pada matriks Hermitian pun berlaku operasi-operasi tersebut, salah satu diantaranya adalah operasi pergandaan skalar. Jika pada matriks Hermitian dikenakan operasi pergandaan skalar $i$ (bilangan imajiner), menghasilkan suatu matriks yang baru, dimana negatif matriks tersebut sama dengan matriks transpose konjugatnya, dan elemen pada diagonal utamanya adalah bilangan imajiner murni. Bentuk matriks baru ini yang dikenal dengan matriks Skew Hermitian.

Selanjutnya dengan memperhatikan elemen diagonal utama beserta elemen transpose konjugatnya, mengakibatkan adanya perbedaan bentuk dan sifat-sifat dari matriks Skew Hermitian jika dibandingkan dengan matriks Hermitian dan matriks lainnya. Hal inilah yang melatarbelakangi peneliti untuk melakukan penelitian dengan judul Sifat-sifat Dasar Matriks Skew Hermitian.

\section{TINJAUAN PUSTAKA}

Matriks adalah suatu konsep dasar dalam dunia Aljabar yang pertama digunakan pada tahun 1850 oleh Sylvester, yang mendefinisikan matriks sebagai susunan 
elemen-elemen dalam bentuk bujursangkar. Kemudian pada tahun 1855 Charles Hermite memperkenalkan matriks Hermitian sebagai bentuk dari matriks bilangan kompleks.

Dengan merujuk pada buku Schaum's Outlines Aljabar Linier Edisi Ketiga, matriks dapat dibedakan menjadi matriks-matriks khusus diantaranya matriks transpose, matriks simetri, matriks simetri miring, dan lain-lain. Selain bentuk-bentuk matriks di atas, dalam jurnal Lecture Notes For Math 623 Matrix Analysis,yang disusun oleh Michael E. O’Sullivan (April 18,2013) ditulis tentang matriks Skew Hermitian dengan keistimewaan dan keunikannya. Berdasarkan sumber tersebut dan dukungan beberapa literatur lainnya peneliti mencoba menyusun sebuah penelitian dengan harapan semoga dapat mudah dipahami.

\section{Definisi 1 (Matriks)}

Matriks didefinisikan sebagai susunan persegi panjang dari bilangan-bilangan yang diatur dalam baris dan kolom. Matriks $A$ ditulis sebagai berikut:

$$
A=\left\|a_{i j}\right\|=\left[\begin{array}{cccc}
a_{11} & a_{12} & \cdots & a_{1 n} \\
a_{21} & a_{22} & \cdots & a_{2 n} \\
\vdots & \vdots & \ddots & \vdots \\
a_{m 1} & a_{m 2} & \cdots & a_{m n}
\end{array}\right]
$$

Susunan di atas disebut sebuah matriks $m$ kali $n$ (ditulis $A_{m \times n}$ ) karena memiliki $m$ baris dan $n$ kolom. Sebagai aturan, kurung siku [ ], kurung biasa ( ) atau bentuk || || digunakan untuk mengurangi susunan persegi panjang dari bilangan-bilangan tersebut.

\section{Teorema 1}

Jika ukuran matriks $A$ dan $B$ adalah sedemikian sehingga operasi matriks dapat dikerjakan, maka
(a) $\left(A^{T}\right)^{T}=A$
(b) $(A+B)^{T}=A^{T}+B^{T}$ dan $(A-B)^{T}=A^{T}-B^{T}$
(c) $(k A)^{T}=k A^{T}, k$ skalar
(d) $(A B)^{T}=B^{T} A^{T}$

Definisi 2 (Matriks Simetris Miring)

Diberikan matriks bujur sangkar $A$ maka matriks simetris miring adalah matriks yang memenuhi:

$$
-A=A^{T}
$$

\section{Contoh 1}

$$
\begin{aligned}
& \mathrm{A}=\left[\begin{array}{ccc}
0 & 3 & -4 \\
-3 & 0 & 5 \\
4 & -5 & 0
\end{array}\right]-A=(-1) A \\
& =(-1)\left[\begin{array}{ccc}
0 & 3 & -4 \\
-3 & 0 & 5 \\
4 & -5 & 0
\end{array}\right] \\
& \quad=\left[\begin{array}{ccc}
0 & -3 & 4 \\
3 & 0 & -5 \\
-4 & 5 & 0
\end{array}\right] \\
& A^{T}=\left[\begin{array}{ccc}
0 & -3 & 4 \\
3 & 0 & -5 \\
-4 & 5 & 0
\end{array}\right]
\end{aligned}
$$

Definisi 3 ( Matriks Hermitian )

Diberikan matriks kompleks bujur sangkar $A$ maka matriks Hermitian adalah matriks yang memenuhi :

$$
A=\overline{A^{T}}
$$

\section{Contoh 2}

$A=\left[\begin{array}{ccc}3 & 1-2 i & 4+7 i \\ 1+2 i & -4 & -2 i \\ 4-7 i & 2 i & 5\end{array}\right]$ dan

$$
\begin{gathered}
\mathrm{A}^{\mathrm{T}}=\left[\begin{array}{ccc}
3 & 1+2 \mathrm{i} & 4-7 \mathrm{i} \\
1-2 \mathrm{i} & -4 & 2 \mathrm{i} \\
4+7 \mathrm{i} & -2 \mathrm{i} & 5
\end{array}\right] \\
\overline{\mathrm{A}^{\mathrm{T}}}=\left[\begin{array}{ccc}
3 & 1-2 \mathrm{l} & 4+7 \mathrm{l} \\
1+2 \mathrm{l} & -4 & -2 \mathrm{l} \\
4-7 \mathrm{l} & 2 \mathrm{l} & 5
\end{array}\right] \\
\overline{\mathrm{A}^{\mathrm{T}}}=\left[\begin{array}{ccc}
3 & 1-2 \mathrm{i} & 4+7 \mathrm{i} \\
1+2 \mathrm{i} & -4 & -2 \mathrm{i} \\
4-7 \mathrm{i} & 2 \mathrm{i} & 5
\end{array}\right]=A
\end{gathered}
$$

Jadi $A=\overline{A^{T}}$

\section{Definisi 4 (Vektor)}

Vektor merupakan besaran yang mempunyai arah dan mempunyai panjang (magnitude) dan diberi notasi dengan huruf tebal, misalnya: $\boldsymbol{v}, \boldsymbol{u}, \boldsymbol{w}$ dan $\boldsymbol{p}$.

Sebuah vektor $\boldsymbol{a}$ berdimensi $n$ adalah suatu aturan $n$ tuple dari bilangan-bilangan ditulis sebagai baris $\left(a_{1}, a_{2}, \ldots, a_{n}\right)$ atau sebagai sebuah kolom

$$
\left[\begin{array}{c}
a_{1} \\
a_{2} \\
\vdots \\
a_{n}
\end{array}\right]
$$

$a_{i}$ merupakan komponen-komponen vektor dimana $a_{i} \in \mathbb{R}$ dan $i=1,2, \ldots, n$.

\section{Definisi 5 (Ruang Vektor)}

Misalkan $\mathrm{V}$ sebarang himpunan dan $V \neq \varnothing$. Himpunan $V$ disebut ruang vektor atas lapangan $L$ terhadap operasi $"+$ " dan ". " yang didefinisikan padanya jika $(\forall u, v, w \in$ $V)(\forall k, l, \in L)$ dipenuhi aksioma-aksioma berikut :
1. $\boldsymbol{u}+\boldsymbol{v} \in \boldsymbol{V}$
2. $(\boldsymbol{u}+\boldsymbol{v})+\boldsymbol{w}=\boldsymbol{u}+(\boldsymbol{v}+w)$
3. $\boldsymbol{u}+0=0+\boldsymbol{u}$
4. $\boldsymbol{u}+(-\boldsymbol{u})=0$
5. $u+v=v+u$
6. $k \boldsymbol{u} \in \boldsymbol{V}$
7. $k(l \boldsymbol{u})=(k l) \boldsymbol{u}$
8. $k(\boldsymbol{u}+\boldsymbol{v})=k \boldsymbol{u}+k \boldsymbol{v}$
9. $(k+l) \boldsymbol{u}=k \boldsymbol{u}+l \boldsymbol{u}$
10. $1 \boldsymbol{u}=\boldsymbol{u}$

Definisi 6 (Bilangan Kompleks)

Sebuah bilangan kompleks $z \in \mathbb{C}$ terdiri atas bagian nyata $x=\operatorname{Re}(z)$ dan bagian imajiner $y=\operatorname{Im}(z)$ dengan bentuk $z=x+i y$. Untuk $i$ sebuah bilangan imajiner $i=\sqrt{-1}$. Untuk selanjutnya bilangan kompleks $z_{n}=x_{n}+i y_{n}$ dengan $n=1,2,3, \ldots$

Definisi 7 (Kesekawanan/Conjugation)

Diberikan $z \in \mathbb{C}, z=x+i y$ maka sekawan $z$ dituliskan sebagai $\bar{z}$ dan didefinisikan sebagai $\bar{z}=x-i y, \bar{z} \in \mathbb{C}$. 
Definisi 8 (Pembagian Bilangan Kompleks)

Diberikan $z_{1}, z_{2} \in \mathbb{C}$, hasil pembagian dua bilangan kompleks berlaku sesuai dengan:

$$
\frac{z_{1}}{z_{2}}=\frac{z_{1}}{z_{2}} \frac{\overline{z_{2}}}{\overline{z_{2}}}
$$

\section{Teorema 2}

Operasi-operasi yang didefinisikan pada bilangan kompleks memenuhi hukum-hukum berikut:

(a) Hukum komutatif

(i). $z_{1}+z_{2}=z_{2}+z_{1}$; ii). $z_{1} z_{2}=z_{2} z_{1}$

(b) Hukum asosiatif

$$
\text { (i). } z_{1}+\left(z_{2}+z_{3}\right)=\left(z_{1}+z_{2}\right)+z_{3} \text {; }
$$$$
\text { (ii). } z_{1}\left(z_{2} z_{3}\right)=\left(z_{1} z_{2}\right) z_{3}
$$

(c) Hukum distributif

(d) Distributivitas kesekawanan

$$
z_{1}\left(z_{2}+z_{3}\right)=\left(z_{1} z_{2}+z_{1} z_{3}\right)
$$

(i). $\overline{z_{1}+z_{2}}=\overline{z_{1}}+\overline{z_{2}}$;

(ii). $\overline{z_{1}-z_{2}}=\overline{z_{1}}-\overline{z_{2}}$;

(iii). $\overline{z_{1} z_{2}}=\overline{z_{1}} \overline{z_{2}}$;

(e) $\overline{(\bar{z})}=z$

(iv). $\overline{z_{1} / z_{2}}=\overline{z_{1}} / \overline{z_{2}}$

(f) $z \bar{z}=(x+i y)(x-i y)$

$$
=x^{2}+y^{2}=(\operatorname{Re}(z))^{2}+
$$

\section{HASIL DAN PEMBAHASAN}

\section{Matriks Skew Hermitian}

\section{Definisi 9}

Sebuah matriks $A \in \mathbb{C}^{n \times n}$ dengan transpose konjugatnya $A^{*}$ disebut Matriks Skew Hermitian jika, $-A=A^{*}$ dengan elemen-elemen penyusunnya merupakan bilangan kompleks, dan semua elemen pada diagonal utama merupakan bilangan imajiner. Elemen pada baris ke- $k$ kolom ke- $l$ sama dengan negatif konjugat kompleks dari elemen pada baris ke- $l$ kolom ke- $k$, atau dengan kata lain untuk setiap $a_{k l}, a_{l k} \in \mathbb{C}$ dan $k, l=$ $1,2, \ldots, n$, berlaku $a_{k l}=\overline{-a}_{l k}$

Matriks Skew Hermitian $A$ dinotasikan dengan:

$$
A=\left\{\left[\begin{array}{cccc}
a_{11} & a_{12} & \cdots & a_{1 n} \\
a_{21} & a_{22} & \cdots & a_{2 n} \\
\vdots & \vdots & & \vdots \\
a_{n 1} & a_{n 2} & \cdots & a_{n n}
\end{array}\right] \mid a_{k l}=-\bar{a}_{l k} \in \mathbb{C}, a_{k k} \in \operatorname{im}(z)\right\}
$$

Himpunan matriks-matriks Skew Hermitian dengan orde $n$ dinotasikan dengan $S H_{n}$.

\section{Contoh 3}

Matriks $A=\left[\begin{array}{cc}-i & 2+i \\ -2+i & 0\end{array}\right]$,

$B=\left[\begin{array}{ccc}i & 2+i & -2+i \\ -2+i & 0 & 3-2 i \\ 2+i & -3-2 i & 0\end{array}\right]$ adalah matriks Skew

Hermitian.

Penyelesaian :

Untuk matriks $A$, dengan mentranspose-konjugatkan matriks $A$ maka diperoleh:

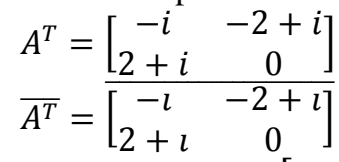

$$
\begin{aligned}
& =\left[\begin{array}{cc}
\overline{-l} & \overline{-2+\imath} \\
\overline{2+\imath} & \overline{0}
\end{array}\right]
\end{aligned}
$$

$$
A^{*}=\left[\begin{array}{cc}
i & -2-i \\
2-i & 0
\end{array}\right]=-A
$$

Karena $A^{*}=-A$, maka $A$ adalah matriks Skew Hermitian.

Untuk matriks $B$, dengan mentranspose-konjugatkan matriks $B$ maka diperoleh:

$$
B^{T}=\left[\begin{array}{ccc}
i & -2+i & 2+i \\
2+i & 0 & -3-2 i \\
-2+i & 3-2 i & 0
\end{array}\right]
$$

$$
\begin{aligned}
& \overline{B^{T}}=\overline{\left[\begin{array}{ccc}
l & -2+\imath & 2+\imath \\
2+\imath & 0 & -3-2 \imath \\
-2+\imath & 3-2 \imath & 0
\end{array}\right]} \\
& =\left[\begin{array}{ccc}
\frac{\bar{\imath}}{2+\imath} & \overline{-2+\imath} & \overline{2+\imath} \\
\overline{-2+\imath} & \overline{0} & \overline{-3-2 \imath} \\
\overline{3-2 \imath} & \overline{0}
\end{array}\right]
\end{aligned}
$$

$$
B^{*}=\left[\begin{array}{ccc}
-i & -2-i & 2-i \\
2-i & 0 & -3+2 i \\
-2-i & 3+2 i & 0
\end{array}\right]=-B
$$

Karena $B^{*}=-B$, maka $B$ adalah Skew Hermitian.

\section{Sifat-Sifat Matriks Skew Hermitian}

\section{Sifat 1}

Untuk sebarang matriks $A \in \mathbb{C}^{n \times n}$ dengan transpose konjugatnya $A^{*} \in \mathbb{C}^{n \times n}$, maka berlaku:

(a) $A+A^{*} \in \mathcal{H}_{n}$

(b) $A-A^{*} \in S H_{n}$

Bukti :

Ambil sebarang $A \in \mathbb{C}^{n \times n}$ dengan transpose konjugatnya $A^{*} \in \mathbb{C}^{n \times n}$

$A=\left[\begin{array}{cccc}a_{11} & a_{12} & \cdots & a_{1 n} \\ a_{21} & a_{22} & \cdots & a_{2 n} \\ \vdots & \vdots & & \vdots \\ a_{n 1} & a_{n 2} & \cdots & a_{n n}\end{array}\right], A^{*}=\left[\begin{array}{cccc}\overline{a_{11}} & \overline{a_{21}} & \cdots & \overline{a_{n 1}} \\ \overline{a_{12}} & \overline{a_{22}} & \cdots & \overline{a_{n 2}} \\ \overline{a_{1 n}} & \overline{a_{2 n}} & \cdots & \overline{a_{n n}}\end{array}\right]$ dimana untuk setiap $a_{k l}, \overline{a_{k l}} \in \mathbb{C}$

dan $a_{k l}=x_{k l}+i y_{k l}, \overline{a_{k l}}=x_{k l}-i y_{k l}$ dengan $k, l=$ $1,2, \ldots, n$.

(a). Dengan menjumlahkan matriks $A \in \mathbb{C}^{n \times n}$ dan $A^{*} \in \mathbb{C}^{n \times n}$ diperoleh:

$$
\begin{gathered}
A+A^{*}=\left[\begin{array}{cccc}
a_{11} & a_{12} & \cdots & a_{1 n} \\
a_{21} & a_{22} & \cdots & a_{2 n} \\
\vdots & \vdots & & \vdots \\
a_{n 1} & a_{n 2} & \cdots & a_{n n}
\end{array}\right]+\left[\begin{array}{cccc}
\overline{a_{11}} & \overline{a_{21}} & \cdots & \overline{a_{n 1}} \\
\overline{a_{12}} & \overline{a_{22}} & \cdots & \overline{a_{n 2}} \\
\vdots & \vdots & & \vdots \\
\overline{a_{1 n}} & \overline{a_{2 n}} & \cdots & \overline{a_{n n}}
\end{array}\right] \\
=\left[\begin{array}{ccc}
x_{11}+y_{11} i & \cdots & x_{1 n}+y_{1 n} i \\
x_{21}+y_{21} i & \cdots & x_{2 n}+y_{2 n} i \\
\vdots & \vdots & \vdots \\
x_{n 1}+y_{n 1} i & \cdots & x_{n n}+y_{n n} i
\end{array}\right] \\
+\left[\begin{array}{cccc}
x_{11}-y_{11} i & \cdots & x_{n 1}-y_{n 1} i \\
x_{12}-y_{12} i & \cdots & x_{n 2}-y_{n 2} i \\
\vdots & \vdots & \vdots \\
x_{1 n}-y_{1 n} i & \cdots & x_{n n}-y_{n n} i
\end{array}\right] \\
=\left[\begin{array}{cccc}
\left(x_{11}+y_{11} i\right)+\left(x_{11}-y_{11} i\right) & \cdots & \left(x_{1 n}+y_{1 n} i\right)+\left(x_{n 1}-y_{n 1} i\right) \\
\left(x_{21}+y_{21} i\right)+\left(x_{12}-y_{12} i\right) & \cdots & \left(x_{2 n}+y_{2 n} i\right)+\left(x_{n 2}-y_{n 2} i\right) \\
\vdots & \vdots & \vdots & \\
\left(x_{n 1}+y_{n 1} i\right)+\left(x_{1 n}-y_{1 n} i\right) & \cdots & \left(x_{n n}+y_{n n} i\right)+\left(x_{n n}-y_{n n} i\right)
\end{array}\right]
\end{gathered}
$$

Dimisalkan $A+A^{*}=B$ maka,

$$
A+A^{*}=B=\left[\begin{array}{cccc}
2 x_{11} & b_{12} & \cdots & b_{1 n} \\
b_{21} & 2 x_{22} & \cdots & b_{2 n} \\
\vdots & \vdots & & \vdots \\
b_{n 1} & b_{n 2} & \cdots & 2 x_{n n}
\end{array}\right]
$$


Dengan,

$\begin{array}{ccc}b_{12}=\left(x_{12}+x_{21}\right)+\left(y_{12}-y_{21}\right) i & \cdots & b_{1 n}=\left(x_{1 n}+x_{n 1}\right)+\left(y_{1 n}-y_{n 1}\right) i \\ b_{21}=\left(x_{21}+x_{12}\right)+\left(y_{21}-y_{12}\right) i & \cdots & b_{2 n=\left(x_{2 n}+x_{n 2}\right)+\left(y_{2 n}-y_{n 2}\right) i} \\ \vdots & & \vdots \\ b_{n 1=\left(x_{n 1}+x_{1 n}\right)+\left(y_{n 1}-y_{1 n}\right) i} & & b_{n 2}=\left(x_{n 2}+x_{2 n}\right)+\left(y_{n 2}-y_{2 n}\right) i\end{array}$

Untuk membuktikan bahwa $A+A^{*}=B$ adalah Hermitian, cukup ditunjukan bahwa $B=B^{*}$.

Dengan mentranspose-konjugatkan matriks $B$ maka diperoleh :

$$
\begin{aligned}
& B^{T}=\left[\begin{array}{cccc}
2 x_{11} & b_{21} & \cdots & b_{n 1} \\
b_{12} & 2 x_{22} & \cdots & b_{n 2} \\
\vdots & \vdots & & \vdots \\
b_{1 n} & b_{2 n} & \cdots & 2 x_{n n}
\end{array}\right] \\
& =\left[\begin{array}{ccc}
2 x_{11} & \cdots & \left(x_{n 1}+x_{1 n}\right)+\left(y_{n 1}-y_{1 n}\right) i \\
\left(x_{12}+x_{21}\right)+\left(y_{12}-y_{21}\right) i & \cdots & \left(x_{n 2}+x_{2 n}\right)+\left(y_{n 2}-y_{2 n}\right) i \\
\vdots & \vdots & \vdots \\
\left(x_{1 n}+x_{n 1}\right)+\left(y_{1 n}-y_{n 1}\right) i & \cdots & 2 x_{n n}
\end{array}\right] \\
& \overline{B^{T}}=\overline{\left[\begin{array}{ccc}
2 x_{11} & \cdots & \left(x_{n 1}+x_{1 n}\right)+\left(y_{n 1}-y_{1 n}\right) \iota \\
\left(x_{12}+x_{21}\right)+\left(y_{12}-y_{21}\right) \iota & \cdots & \left(x_{n 2}+x_{2 n}\right)+\left(y_{n 2}-y_{2 n}\right) \iota \\
\vdots & \vdots & \vdots \\
\left(x_{1 n}+x_{n 1}\right)+\left(y_{1 n}-y_{n 1}\right) \iota & \cdots & 2 x_{n n}
\end{array}\right]} \\
& =\left[\begin{array}{ccc}
\overline{2 x_{11}} & \cdots & \overline{\left(x_{n 1}+x_{1 n}\right)+\left(y_{n 1}-y_{1 n}\right) \iota} \\
\frac{\left.1 x_{12}+x_{21}\right)+\left(y_{12}-y_{21}\right) \iota}{x_{n 2}} & \cdots & \overline{\left(x_{n 2}+x_{2 n}\right)+\left(y_{n 2}-y_{2 n}\right) \iota} \\
\frac{\vdots}{\left(x_{1 n}+x_{n 1}\right)+\left(y_{1 n}-y_{n 1}\right) \imath} & \cdots & \vdots \\
2 x_{n n}
\end{array}\right] \\
& =\left[\begin{array}{ccc}
2 x_{11} & \cdots & \left(x_{n 1}+x_{1 n}\right)-\left(y_{n 1}-y_{1 n}\right) i \\
\left(x_{12}+x_{21}\right)-\left(y_{12}-y_{21}\right) i & \cdots & \left(x_{n 2}+x_{2 n}\right)-\left(y_{n 2}-y_{2 n}\right) i \\
\vdots & \vdots & \vdots \\
\left(x_{1 n}+x_{n 1}\right)-\left(y_{1 n}-y_{n 1}\right) i & \cdots & 2 x_{n n}
\end{array}\right]
\end{aligned}
$$$$
\overline{B^{T}}=B^{*}
$$$$
=\left[\begin{array}{ccc}
2 x_{11} & \cdots & \left(x_{1 n}+x_{n 1}\right)+\left(y_{1 n}-y_{n 1}\right) i \\
\left(x_{21}+x_{12}\right)+\left(y_{21}-y_{12}\right) i & \cdots & \left(x_{2 n}+x_{n 2}\right)+\left(y_{2 n}-y_{n 2}\right) i \\
\vdots & \vdots & \vdots \\
\left(x_{n 1}+x_{1 n}\right)+\left(y_{n 1}-y_{1 n}\right) i & \cdots & 2 x_{n n}
\end{array}\right]
$$$$
B^{*}=\left[\begin{array}{cccc}
2 x_{11} & b_{12} & \cdots & b_{1 n} \\
b_{21} & 2 x_{22} & \cdots & b_{2 n} \\
\vdots & \vdots & & \vdots \\
b_{n 1} & b_{n 2} & \cdots & 2 x_{n n}
\end{array}\right]=B
$$

Dengan,

$$
\begin{array}{ccc}
b_{12}=\left(x_{12}+x_{21}\right)+\left(y_{12}-y_{21}\right) i & \cdots & b_{1 n}=\left(x_{1 n}+x_{n 1}\right)+\left(y_{1 n}-y_{n 1}\right) i \\
b_{21}=\left(x_{21}+x_{12}\right)+\left(y_{21}-y_{12}\right) i & \cdots & b_{2 n}=\left(x_{2 n}+x_{n 2}\right)+\left(y_{2 n}-y_{n 2}\right) i \\
\vdots & & \vdots \\
b_{n 1}=\left(x_{n 1}+x_{1 n}\right)+\left(y_{n 1}-y_{1 n}\right) i & & b_{n 2}=\left(x_{n 2}+x_{2 n}\right)+\left(y_{n 2}-y_{2 n}\right) i
\end{array}
$$

Karna $B=B^{*}$, maka $A+A^{*}=B$ adalah Hermitian.

(b). Dengan mengurangkan matriks $A \in \mathbb{C}^{n \times n}$ dan $A^{*} \in \mathbb{C}^{n \times n}$ diperoleh :

$$
\begin{aligned}
A & A^{*}=\left[\begin{array}{cccc}
a_{11} & a_{12} & \cdots & a_{1 n} \\
a_{21} & a_{22} & \cdots & a_{2 n} \\
\vdots & \vdots & & \vdots \\
a_{n 1} & a_{n 2} & \cdots & a_{n n}
\end{array}\right]-\left[\begin{array}{cccc}
\overline{a_{11}} & \overline{a_{21}} & \cdots & \overline{a_{n 1}} \\
\overline{a_{12}} & \overline{a_{22}} & \cdots & \overline{a_{n 2}} \\
\vdots & \vdots & & \vdots \\
\overline{a_{1 n}} & \overline{a_{2 n}} & \cdots & \overline{a_{n n}}
\end{array}\right] \\
& =\left[\begin{array}{ccc}
x_{11}+y_{11} i & \cdots & x_{1 n}+y_{1 n} i \\
x_{21}+y_{21} i & \cdots & x_{2 n}+y_{2 n} i \\
\vdots & \vdots & \vdots \\
x_{n 1}+y_{n 1} i & \cdots & x_{n n}+y_{n n} i
\end{array}\right]-\left[\begin{array}{cccc}
x_{11}-y_{11} i & \cdots & x_{n 1}-y_{n 1} i \\
x_{12}-y_{12} i & \cdots & x_{n 2}-y_{n 2} i \\
\vdots & \vdots & \vdots \\
x_{1 n}-y_{1 n} i & \cdots & x_{n n}-y_{n n} i
\end{array}\right] \\
& =\left[\begin{array}{cccc}
\left(x_{11}+y_{11} i\right)-\left(x_{11}-y_{11} i\right) & \cdots & \left(x_{1 n}+y_{1 n} i\right)-\left(x_{n 1}-y_{n 1} i\right) \\
\left(x_{21}+y_{21} i\right)-\left(x_{12}-y_{12} i\right) & \cdots & \left(x_{2 n}+y_{2 n} i\right)-\left(x_{n 2}-y_{n 2} i\right) \\
\vdots & \vdots & \vdots \\
\left(x_{n 1}+y_{n 1} i\right)-\left(x_{1 n}-y_{1 n} i\right) & \cdots & \left(x_{n n}+y_{n n} i\right)-\left(x_{n n}-y_{n n} i\right)
\end{array}\right]
\end{aligned}
$$

Dimisalkan $A-A^{*}=C$ maka

$$
A-A^{*}=C=\left[\begin{array}{cccc}
2 y_{11} i & c_{12} & \cdots & c_{1 n} \\
c_{21} & 2 y_{22} i & \cdots & c_{2 n} \\
\vdots & \vdots & & \vdots \\
c_{n 1} & c_{n 2} & \cdots & 2 y_{n n} i
\end{array}\right]
$$

Dengan,

$$
\begin{array}{ccc}
c_{12}=\left(x_{12}-x_{21}\right)+\left(y_{12}+y_{21}\right) i & \cdots & c_{1 n}=\left(x_{1 n}-x_{n 1}\right)+\left(y_{1 n}+y_{n 1}\right) i \\
c_{21}=\left(x_{21}-x_{12}\right)+\left(y_{21}+y_{12}\right) i & \cdots & c_{2 n}=\left(x_{2 n}-x_{n 2}\right)+\left(y_{2 n}+y_{n 2}\right) i \\
\vdots & & \vdots \\
c_{n 1}=\left(x_{n 1}-x_{1 n}\right)+\left(y_{n 1}+y_{1 n}\right) i & & c_{n 2}=\left(x_{n 2}-x_{2 n}\right)+\left(y_{n 2}+y_{2 n}\right) i
\end{array}
$$

Untuk membuktikan bahwa $A-A^{*}=C$ adalah Skew Hermitian, cukup ditunjukan bahwa $-C=C^{*}$.

Dengan mentranspose-konjugatkan matriks $C$ maka diperoleh :

$$
\begin{aligned}
& C^{T}=\left[\begin{array}{cccc}
2 y_{11 i} & c_{21} & \cdots & c_{n 1} \\
c_{12} & 2 y_{22 i} & \cdots & c_{n 2} \\
\vdots & \vdots & & \vdots \\
c_{1 n} & c_{2 n} & \cdots & 2 y_{n n i}
\end{array}\right] \\
& =\left[\begin{array}{ccc}
2 y_{11 i} & \cdots & \left(x_{n 1}-x_{1 n}\right)+\left(y_{n 1}+y_{1 n}\right) i \\
\left(x_{12}-x_{21}\right)+\left(y_{12}+y_{21}\right) i & \cdots & \left(x_{n 2}-x_{2 n}\right)+\left(y_{n 2}+y_{2 n}\right) i \\
\vdots & \vdots & \vdots \\
\left(x_{1 n}-x_{n 1}\right)+\left(y_{1 n}+y_{n 1}\right) i & \cdots & 2 y_{n n i}
\end{array}\right] \\
& \left.=\begin{array}{ccc}
2 y_{11 l} & \cdots & \left(x_{n 1}-x_{1 n}\right)+\left(y_{n 1}+y_{1 n}\right) l \\
\left(x_{12}-x_{21}\right)+\left(y_{12}+y_{21}\right) \iota & \cdots & \left(x_{n 2}-x_{2 n}\right)+\left(y_{n 2}+y_{2 n}\right) \iota \\
\vdots & \vdots & \vdots \\
\left(x_{1 n}-x_{n 1}\right)+\left(y_{1 n}+y_{n 1}\right) \iota & \cdots & 2 y_{n n l}
\end{array}\right] \\
& =\left[\begin{array}{ccc}
\overline{2 y_{11 \imath}} & \cdots & \overline{\left(x_{n 1}-x_{1 n}\right)+\left(y_{n 1}+y_{1 n}\right) \iota} \\
\overline{\left(x_{12}-x_{21}\right)+\left(y_{12}+y_{21}\right) \iota} & \cdots & \overline{\left(x_{n 2}-x_{2 n}\right)+\left(y_{n 2}+y_{2 n}\right) \iota} \\
\vdots & \vdots & \vdots \\
\frac{2 y_{n n \imath}}{\left(x_{1 n}-x_{n 1}\right)+\left(y_{1 n}+y_{n 1}\right) \iota} & \cdots & \cdots
\end{array}\right] \\
& =\left[\begin{array}{ccc}
-2 y_{11 i} & \cdots & \left(x_{n 1}-x_{1 n}\right)-\left(y_{n 1}+y_{1 n}\right) i \\
\left(x_{12}-x_{21}\right)-\left(y_{12}+y_{21}\right) i & \cdots & \left(x_{n 2}-x_{2 n}\right)-\left(y_{n 2}+y_{2 n}\right) i \\
\vdots & \vdots & \vdots \\
\left(x_{1 n}-x_{n 1}\right)-\left(y_{1 n}+y_{n 1}\right) i & \cdots & -2 y_{n n i}
\end{array}\right]
\end{aligned}
$$

$\overline{C^{T}}=C^{*}$

$$
\begin{gathered}
=\left[\begin{array}{cccc}
-2 y_{11 i} & \cdots & \left(-x_{1 n}+x_{n 1}\right)-\left(y_{1 n}+y_{n 1}\right) i \\
\left(-x_{21}+x_{12}\right)-\left(y_{21}+y_{12}\right) i & \cdots & \left(-x_{2 n}+x_{n 2}\right)-\left(y_{2 n}+y_{n 2}\right) i \\
\vdots & \vdots & & \vdots \\
\left(-x_{n 1}+x_{1 n}\right)-\left(y_{n 1}+y_{1 n}\right) i & \cdots & & -2 y_{n n i}
\end{array}\right] \\
C^{*}=\left[\begin{array}{cccc}
-2 y_{11} i & c_{12} & \cdots & c_{1 n} \\
c_{21} & -2 y_{22} i & \cdots & c_{2 n} \\
\vdots & \vdots & & \vdots \\
c_{n 1} & c_{n 2} & \cdots & -2 y_{n n} i
\end{array}\right]=-C
\end{gathered}
$$

Dengan,

$$
\begin{array}{ccc}
c_{12}=\left(-x_{12}+x_{21}\right)-\left(y_{12}+y_{21}\right) i & \cdots & c_{1 n=\left(-x_{1 n}+x_{n 1}\right)-\left(y_{1 n}+y_{n 1}\right) i} \\
c_{21}=\left(-x_{21}+x_{12}\right)-\left(y_{21}+y_{12}\right) i & \cdots & c_{2 n=\left(-x_{2 n}+x_{n 2}\right)-\left(y_{2 n}+y_{n 2}\right) i} \\
\vdots & & \vdots \\
c_{n 1}=\left(-x_{n 1}+x_{1 n}\right)-\left(y_{n 1}+y_{1 n}\right) i & & c_{n 2}=\left(-x_{n 2}+x_{2 n}\right)-\left(y_{n 2}+y_{2 n}\right) i
\end{array}
$$

Terlihat bahwa $-C=C^{*}$. Karna $-C=C^{*}$, maka $A-A^{*}=C$ adalah Skew Hermitian.

\section{Sifat 2}

Suatu matriks $A \in \mathbb{C}^{n \times n}$, dapat dituliskan sebagai jumlah dari matriks Hermitian dan matriks Skew Hermitian

Bukti :

$$
A=\frac{1}{2}\left(A+A^{*}\right)+\frac{1}{2}\left(A-A^{*}\right)
$$

Dari sifat 1 diketahui bahwa

$$
A+A^{*} \in H_{n}, \operatorname{dan} A-A^{*} \in S H_{n}
$$




$$
\begin{aligned}
& \text { Sehingga } \\
& \frac{1}{2}\left(A+A^{*}\right)+\frac{1}{2}\left(A-A^{*}\right)=\frac{1}{2}\left(\left(A+A^{*}\right)+\left(A-A^{*}\right)\right) \\
& =\frac{1}{2}\left((A+A)+\left(A^{*}-A^{*}\right)\right) \\
& =\frac{1}{2}(2 A) \\
& =\left(\frac{1}{2} \cdot 2\right) A \\
& =A
\end{aligned}
$$

\section{Sifat 3}

$A$ adalah Hermitian jika dan hanya jika $i A$ adalah Skew Hermitian

\section{Bukti :}

$(\Leftarrow)$ Diketahui $i A$ Skew Hermitian.

Akan ditunjukan $A$ adalah Hermitian.

Ambil sebarang $A \in \mathcal{S} H_{n}$

Sehingga

$$
A=\left[\begin{array}{cccc}
y_{11} i & a_{12} & \cdots & a_{1 n} \\
a_{21} & y_{22} i & \cdots & a_{2 n} \\
\vdots & \vdots & & \vdots \\
a_{n 1} & a_{n 2} & \cdots & y_{n n} i
\end{array}\right]
$$

$$
\begin{aligned}
i A & =i\left[\begin{array}{cccc}
y_{11} i & a_{12} & \cdots & a_{1 n} \\
a_{21} & y_{22} i & \cdots & a_{2 n} \\
\vdots & \vdots & & \vdots \\
a_{n 1} & a_{n 2} & \cdots & y_{n n} i
\end{array}\right] \\
& =\left[\begin{array}{cccc}
y_{11} i^{2} & a_{12} i & \cdots & a_{1 n} i \\
a_{21} i & y_{22} i^{2} & \cdots & a_{2 n} i \\
\vdots & \vdots & & \vdots \\
a_{n 1} i & a_{n 2} i & \cdots & y_{n n} i^{2}
\end{array}\right]
\end{aligned}
$$

Dimisalkan $i A=B$ maka,

$$
i A=B=\left[\begin{array}{cccc}
-y_{11} & b_{12} & \cdots & b_{1 n} \\
b_{21} & -y_{22} & \cdots & b_{2 n} \\
\vdots & \vdots & & \vdots \\
b_{n 1} & b_{n 2} & \cdots & -y_{n n}
\end{array}\right]
$$

Untuk membuktikan bahwa $i A=B$ adalah Hermitian cukup ditunjukan bahwa $B=B^{*}, b_{k l}=$ $\bar{b}_{l k}, b_{k k} \in \operatorname{Re}(z)$

Dengan mentranspose-konjugatkan matriks $B$ maka diperoleh :

$$
\begin{aligned}
B^{T} & =\left[\begin{array}{cccc}
-y_{11} & b_{21} & \cdots & b_{n 1} \\
b_{12} & -y_{22} & \cdots & b_{n 2} \\
\vdots & \vdots & & \vdots \\
b_{1 n} & b_{2 n} & \cdots & -y_{n n}
\end{array}\right] \\
\overline{B^{T}} & =\left[\begin{array}{cccc}
-y_{11} & b_{21} & \cdots & b_{n 1} \\
b_{12} & -y_{22} & \cdots & b_{n 2} \\
\vdots & \vdots & & \vdots \\
b_{1 n} & b_{2 n} & \cdots & -y_{n n}
\end{array}\right] \\
& =\left[\begin{array}{cccc}
-y_{11} & \overline{b_{21}} & \cdots & \overline{b_{n 1}} \\
\overline{b_{12}} & \frac{-y_{22}}{b_{n 2}} & \cdots & \overline{b_{n 2}} \\
\vdots & \vdots & & \vdots \\
\overline{b_{1 n}} & \frac{b_{2 n}}{b_{2 n}} & \cdots & \frac{-y_{n n}}{n}
\end{array}\right.
\end{aligned}
$$

$$
=\left[\begin{array}{cccc}
-y_{11} & \overline{b_{21}} & \cdots & \overline{b_{n 1}} \\
\overline{b_{12}} & -y_{22} & \cdots & \overline{b_{n 2}} \\
\vdots & \vdots & & \vdots \\
\overline{b_{1 n}} & \overline{b_{2 n}} & \cdots & -y_{n n}
\end{array}\right]
$$

Terlihat bahwa $b_{k k} \in \operatorname{Re}(z)$, dan karena $b_{k l}=\bar{b}_{l k}$, maka matriks $B^{*}$ dapat ditulis menjadi

$$
B^{*}=\left[\begin{array}{cccc}
-y_{11} & b_{12} & \cdots & b_{1 n} \\
b_{21} & -y_{22} & \cdots & b_{2 n} \\
\vdots & \vdots & & \vdots \\
b_{n 1} & b_{n 2} & \cdots & -2 y_{n n}
\end{array}\right]=B
$$

Karena $B=B^{*}$ maka $B$ adalah Hermitian.

\section{$(\Rightarrow)$ Diketahui $A$ Hermitian .}

Akan ditunjukan bahwa $i A$ adalah Skew Hermitian.

Ambil sebarang $A \in \mathcal{H}_{n}$

Sehingga

$$
A=\left[\begin{array}{cccc}
x_{11} & a_{12} & \cdots & a_{1 n} \\
a_{21} & x_{22} & \cdots & a_{2 n} \\
\vdots & \vdots & & \vdots \\
a_{n 1} & a_{n 2} & \cdots & x_{n n}
\end{array}\right]
$$

$$
\begin{aligned}
i A & =i\left[\begin{array}{cccc}
x_{11} & a_{12} & \cdots & a_{1 n} \\
a_{21} & x_{22} & \cdots & a_{2 n} \\
\vdots & \vdots & & \vdots \\
a_{n 1} & a_{n 2} & \cdots & x_{n n}
\end{array}\right] \\
& =\left[\begin{array}{cccc}
x_{11} i & a_{12} i & \cdots & a_{1 n} i \\
a_{21} i & x_{22} i & \cdots & a_{2 n} i \\
\vdots & \vdots & & \vdots \\
a_{n 1} i & a_{n 2} i & \cdots & x_{n n} i
\end{array}\right]
\end{aligned}
$$

Dimisalkan $i A=C$ maka,

$$
i A=C=\left[\begin{array}{cccc}
y_{11} i & c_{12} & \cdots & c_{1 n} \\
c_{21} & y_{22} i & \cdots & c_{2 n} \\
\vdots & \vdots & & \vdots \\
c_{n 1} & c_{n 2} & \cdots & y_{n n i}
\end{array}\right]
$$

Untuk membuktikan bahwa $i A=C$ adalah Skew hermitian cukup ditunjukan bahwa $C=-C^{*}$, $c_{k l}=\overline{-c}_{l k}, c_{k k} \in \operatorname{Im}(z)$

Untuk matriks $C$, dengan mentranspose-konjugatkan matriks $C$ maka diperoleh :

$$
\begin{aligned}
& C^{T}=\left[\begin{array}{cccc}
y_{11} i & c_{21} & \cdots & c_{n 1} \\
c_{12} & y_{22} i & \cdots & c_{n 2} \\
\vdots & \vdots & & \vdots \\
c_{1 n} & c_{2 n} & \cdots & y_{n n} i
\end{array}\right] \\
& \overline{C^{T}}=\left[\begin{array}{cccc}
y_{11} l & c_{21} & \cdots & c_{n 1} \\
c_{12} & y_{22} l & \cdots & c_{n 2} \\
\vdots & \vdots & & \vdots \\
c_{1 n} & c_{2 n} & \cdots & y_{n n} l
\end{array}\right] \\
& =\left[\begin{array}{cccc}
\overline{c_{1 n}} & \frac{c_{2 n}}{c_{21}} & \ldots & \frac{y_{n n} l}{c_{n 1}} \\
\overline{c_{12}} & \overline{y_{22} l} & \ldots & \overline{c_{n 2}} \\
\vdots & \vdots & & \vdots \\
\overline{c_{1 n}} & \overline{c_{2 n}} & \ldots & \overline{y_{n n} l}
\end{array}\right] \\
& =\left[\begin{array}{cccc}
-y_{11} i & \overline{c_{21}} & \cdots & \overline{c_{n 1}} \\
\overline{c_{12}} & -y_{22} i & \cdots & \overline{c_{n 2}} \\
\vdots & \vdots & & \vdots \\
\overline{c_{1 n}} & \overline{c_{2 n}} & \cdots & -y_{n n} i
\end{array}\right] \\
& -C^{*}=\left[\begin{array}{cccc}
\frac{y_{11} i}{-c_{12}} & \overline{-c_{21}} & \cdots & \overline{-c_{n 1}} \\
\vdots & \vdots & \cdots & \overline{-c_{n 2}} \\
\overline{-c_{1 n}} & \overline{-c_{2 n}} & \cdots & y_{n n} i
\end{array}\right]
\end{aligned}
$$


Terlihat bahwa $c_{k k} \in \operatorname{Im}(z)$, dan karena $c_{k l}=-\bar{c}_{l k}$, maka matriks $-C^{*}$ dapat ditulis menjadi

$$
-C^{*}=\left[\begin{array}{cccc}
y_{11} i & c_{12} & \cdots & c_{1 n} \\
c_{21} & y_{22} i & \cdots & c_{2 n} \\
\vdots & \vdots & & \vdots \\
c_{n 1} & c_{n 2} & \cdots & y_{n n} i
\end{array}\right]=C
$$

Karena $C=-C^{*}$, maka $C$ adalah Skew hermitian. $\operatorname{Dari}(\Leftarrow)$ dan $(\Leftarrow)$ maka sifat 3 terbukti.

\section{Sifat 4}

Diberikan $A, B$ matriks Skew Hermitian

a) $A+B$ adalah Skew Hermitian

b) Jika $A B=B A$ (berlaku sifat komutatif) maka $A B$ adalah Skew Hermitian

c) Jika $c \in \mathcal{R}$, maka $c A$ adalah Skew Hermitian

Bukti :

Diketahui $A, B$ adalah matriks Skew Hermitian.

Akan ditunjukan:

a) $A+B$ adalah matriks Skew Hermitian

Ambil sebarang matriks $A, B \in \mathcal{S} H_{n}$

Dengan,

$A=\left[\begin{array}{cccc}\alpha_{11} i & a_{12} & \cdots & a_{1 n} \\ a_{21} & \alpha_{22} i & \cdots & a_{2 n} \\ \vdots & \vdots & & \vdots \\ a_{n 1} & a_{n 2} & \cdots & \alpha_{n n} i\end{array}\right], B=\left[\begin{array}{cccc}\beta_{11} i & b_{12} & \cdots & b_{1 n} \\ b_{21} & \beta_{22} i & \cdots & b_{2 n} \\ \vdots & \vdots & & \vdots \\ b_{n 1} & b_{n 2} & \cdots & \beta_{n n} i\end{array}\right]$

Maka

$A+B=\left[\begin{array}{cccc}\alpha_{11} i & a_{12} & \cdots & a_{1 n} \\ a_{21} & \alpha_{22} i & \cdots & a_{2 n} \\ \vdots & \vdots & & \vdots \\ a_{n 1} & a_{n 2} & \cdots & \alpha_{n n} i\end{array}\right]+\left[\begin{array}{cccc}\beta_{11} i & b_{12} & \cdots & b_{1 n} \\ b_{21} & \beta_{22} i & \cdots & b_{2 n} \\ \vdots & \vdots & & \vdots \\ b_{n 1} & b_{n 2} & \cdots & \beta_{n n} i\end{array}\right]$

$$
=\left[\begin{array}{cccc}
\alpha_{11} i+\beta_{11} i & a_{12}+b_{12} & \cdots & a_{1 n}+b_{1 n} \\
a_{21}+b_{21} & \alpha_{22} i+\beta_{22} i & \cdots & a_{2 n}+b_{2 n} \\
\vdots & \vdots & & \vdots \\
a_{n 1}+b_{n 1} & a_{n 2}+b_{n 2} & \cdots & \alpha_{n n} i+\beta_{n n} i
\end{array}\right]
$$

Dimisalkan $A+B=C$ maka,

$$
C=\left[\begin{array}{cccc}
\gamma_{11} i & c_{12} & \cdots & c_{1 n} \\
c_{21} & \gamma_{22} i & \cdots & c_{2 n} \\
\vdots & \vdots & & \vdots \\
c_{n 1} & c_{n 2} & \cdots & \gamma_{n n} i
\end{array}\right]
$$

Untuk membuktikan bahwa $A+B=C$ adalah Skew

Hermitian, cukup ditunjukan bahwa $C=-C^{*}$

Dengan mentranspose-konjugatkan matriks $C$ diperoleh:

$$
\begin{aligned}
C^{T} & =\left[\begin{array}{cccc}
\gamma_{11} i & c_{21} & \cdots & c_{n 1} \\
c_{12} & \gamma_{22} i & \cdots & c_{n 2} \\
\vdots & \vdots & & \vdots \\
c_{1 n} & c_{2 n} & \cdots & \gamma_{n n} i
\end{array}\right] \\
\overline{C^{T}} & =\left[\begin{array}{cccc}
\gamma_{11} l & c_{21} & \cdots & c_{n 1} \\
c_{12} & \gamma_{22} l & \cdots & c_{n 2} \\
\vdots & \vdots & & \vdots \\
c_{1 n} & c_{2 n} & \cdots & \gamma_{n n} l
\end{array}\right] \\
& =\left[\begin{array}{cccc}
\overline{\gamma_{11} l} & \overline{c_{21}} & \cdots & \overline{c_{n 1}} \\
\overline{c_{12}} & \overline{\gamma_{22} l} & \cdots & \overline{c_{n 2}} \\
\vdots & \vdots & & \vdots \\
\overline{c_{1 n}} & \frac{c_{2 n}}{c_{2 n}} & \cdots & \overline{\gamma_{n n} l}
\end{array}\right]
\end{aligned}
$$

$$
\begin{array}{r}
=\left[\begin{array}{ccccc}
-\gamma_{11} i & \overline{c_{21}} & \cdots & \overline{c_{n 1}} \\
\overline{c_{12}} & -\gamma_{22} i & \cdots & \overline{c_{n 2}} \\
\vdots & \vdots & & \vdots \\
\overline{c_{1 n}} & \overline{c_{2 n}} & & \cdots & -\gamma_{n n} i
\end{array}\right] \\
-C^{*}=\left[\begin{array}{cccc}
\gamma_{11} i & \overline{-c_{21}} & \cdots & \overline{-c_{n 1}} \\
-\overline{c_{12}} & \gamma_{22} i & \cdots & -\overline{c_{n 2}} \\
\vdots & \vdots & & \vdots \\
\overline{-c_{1 n}} & -\overline{c_{2 n}} & \cdots & \gamma_{n n} i
\end{array}\right]
\end{array}
$$

Karena $c_{k l}=\overline{-}_{l k}, c_{k k} \in \operatorname{Im}(z)$ maka matriks $-C^{*}$ dapat ditulis menjadi

$$
-C^{*}=\left[\begin{array}{cccc}
\gamma_{11} i & c_{12} & \cdots & c_{1 n} \\
c_{21} & \gamma_{22} i & \cdots & c_{2 n} \\
\vdots & \vdots & & \vdots \\
c_{n 1} & c_{n 2} & \cdots & \gamma_{n n} i
\end{array}\right]=C
$$

Karna $C=-C^{*}$, maka $A+B=C$ adalah Skew Hermitian

b) Jika $A B=B A$ (berlaku sifat komutatif) maka $A B$ adalah Skew Hermitian.

Ambil sebarang $A, B \in \mathcal{S} H_{n}$

$$
\begin{aligned}
A=\left[\begin{array}{cccc}
\alpha_{11} i & a_{12} & \cdots & a_{1 n} \\
a_{21} & \alpha_{22} i & \cdots & a_{2 n} \\
\vdots & \vdots & & \vdots \\
a_{n 1} & a_{n 2} & \cdots & \alpha_{n n} i
\end{array}\right], B & \\
& =\left[\begin{array}{cccc}
\beta_{11} i & b_{12} & \cdots & b_{1 n} \\
b_{21} & \beta_{22} i & \cdots & b_{2 n} \\
\vdots & \vdots & & \vdots \\
b_{n 1} & b_{n 2} & \cdots & \beta_{n n} i
\end{array}\right]
\end{aligned}
$$$$
A B=\left[\begin{array}{cccc}
\alpha_{11} i & a_{12} & \cdots & a_{1 n} \\
a_{21} & \alpha_{22} i & \cdots & a_{2 n} \\
\vdots & \vdots & & \vdots \\
a_{n 1} & a_{n 2} & \cdots & \alpha_{n n} i
\end{array}\right]\left[\begin{array}{cccc}
\beta_{11} i & b_{12} & \cdots & b_{1 n} \\
b_{21} & \beta_{22} i & \cdots & b_{2 n} \\
\vdots & \vdots & & \vdots \\
b_{n 1} & b_{n 2} & \cdots & \beta_{n n} i
\end{array}\right]
$$$$
=\left[\begin{array}{ccc}
\alpha_{11} i \beta_{11} i+\cdots+a_{1 n} b_{n 1} & \cdots & \alpha_{11} i b_{1 n}+\cdots+a_{1 n} \beta_{n n} i \\
a_{21} \beta_{11} i+\cdots+a_{2 n} b_{n 1} & \cdots & a_{21} b_{1 n}+\cdots+a_{2 n} \beta_{n n} i \\
\vdots & \vdots & \vdots \\
a_{n 1} \beta_{11} i+\cdots+\alpha_{n n} i b_{n 1} & \cdots & a_{n 1} b_{1 n}+\cdots+\alpha_{n n} i \beta_{n n} i
\end{array}\right]
$$

Jika hasil kali $A B n \times n$ matriks $C$, maka

$$
C_{i j}=\sum_{k=1}^{n} a_{i k} b_{k j} i=1,2, \ldots, n j=1,2, \ldots, n
$$

Sehingga $\sum_{k=1}^{n} a_{i k} b_{k j} \in \mathbb{C} \quad$ (sifat tertutup terhadap pergandaan pada bilangan kompleks).

Dengan cara yang sama diperoleh:

$$
\begin{aligned}
B A & =\left[\begin{array}{cccc}
\beta_{11} i & b_{12} & \cdots & b_{1 n} \\
b_{21} & \beta_{22} i & \cdots & b_{2 n} \\
\vdots & \vdots & & \vdots \\
b_{n 1} & b_{n 2} & \cdots & \beta_{n n} i
\end{array}\right]\left[\begin{array}{cccc}
y_{11} i & a_{12} & \cdots & a_{1 n} \\
a_{21} & y_{22} i & \cdots & a_{2 n} \\
\vdots & \vdots & & \vdots \\
a_{n 1} & a_{n 2} & \cdots & y_{n n} i
\end{array}\right] \\
& =\left[\begin{array}{cccc}
\beta_{11} i \alpha_{11} i+\cdots+b_{1 n} a_{n 1} & \cdots & \beta_{11} i a_{1 n}+\cdots+b_{1 n} \alpha_{n n} i \\
b_{21} \alpha_{11} i+\cdots+b_{2 n} a_{n 1} & \cdots & b_{21} a_{1 n}+\cdots+b_{2 n} \alpha_{n n} i \\
\vdots & \vdots & \vdots \\
b_{n 1} \alpha_{11} i+\cdots+\beta_{n n} i a_{n 1} & \cdots & b_{n 1} a_{1 n}+\cdots+\beta_{n n} i \alpha_{n n} i
\end{array}\right]
\end{aligned}
$$

Jika hasil kali $B A n \times n$ matriks $D$, maka

$$
D_{i j}=\sum_{k=1}^{n} a_{i k} b_{k j} i=1,2, \ldots, n j=1,2, \ldots, n
$$


Sehingga

$$
\sum_{k=1}^{n} b_{i k} a_{k j} \in \mathbb{C}
$$

(sifat tertutup terhadap pergandaan pada bilangan kompleks).

Dari kedua hasil kali matriks di atas, elemen-elemen penyusunnya haruslah merupakan bilangan kompleks dengan negatif transpose konjugatnya. Untuk sebarang $a_{i k} b_{k j} \in \mathbb{C}$ berlaku:

$$
\begin{aligned}
C_{i j}=\sum_{k=1}^{n} a_{i k} b_{k j} & =a_{i 1} b_{1 j}+a_{i 2} b_{2 j}+\cdots+a_{i n} b_{n j} \\
& =-a_{i 1} b_{1 j}-a_{i 2} b_{2 j}-\cdots-a_{i n} b_{n j} \\
& =\bar{a}_{1 i} \bar{b}_{j 1}-\bar{a}_{2 i} \bar{b}_{j 2}-\cdots-\bar{a}_{n i} \bar{b}_{j n} \\
& =-\bar{b}_{j 1} \bar{a}_{1 i}-\bar{b}_{j 2} \bar{a}_{2 i}-\cdots-\bar{b}_{j n} \bar{a}_{n i} \\
& =(-1)\left(\bar{b}_{j 1} \bar{a}_{1 i}+\bar{b}_{j 2} \bar{a}_{2 i}+\cdots+\bar{b}_{j n} \bar{a}_{n i}\right) \\
& =(-1) \sum_{k=1}^{n} \bar{b}_{j k} \bar{a}_{k i}
\end{aligned}
$$

Karena $A B=B A$, diperoleh:

$$
\begin{aligned}
C_{i j} & =\sum_{k=1}^{n} a_{i k} b_{k j}=D_{i j}=\sum_{k=1}^{n} b_{i k} a_{k j} \\
& (-1) \sum_{k=1}^{n} \bar{b}_{j k} \bar{a}_{k i}=(-1) \sum_{k=1}^{n} \bar{a}_{j k} \bar{b}_{k i}
\end{aligned}
$$

dari kedua persamaan diatas diperoleh:

$$
\begin{gathered}
\sum_{k=1}^{n} a_{i k} b_{k j}=\sum_{k=1}^{n} b_{i k} a_{k j}=(-1) \sum_{k=1}^{n} \bar{b}_{j k} \bar{a}_{k i} \\
=(-1) \sum_{k=1}^{n} \bar{a}_{j k} \bar{b}_{k i}
\end{gathered}
$$

Karena elemen-elemen penyusunnya merupakan bilangan kompleks dengan negatif transpose konjugatnya, maka $A B=B A$ juga merupakan matriks Skew Hermitian.

c) Ambil sebarang $A \in \mathcal{S} H_{n}$. dan $c$ skalar

$$
\begin{aligned}
c A=c & {\left[\begin{array}{cccc}
y_{11} i & a_{12} & \cdots & a_{1 n} \\
a_{21} & y_{22} i & \cdots & a_{2 n} \\
\vdots & \vdots & & \vdots \\
a_{n 1} & a_{n 2} & \cdots & y_{n n} i
\end{array}\right] } \\
& =\left[\begin{array}{cccc}
c y_{11} i & c a_{12} & \cdots & c a_{1 n} \\
c a_{21} & c y_{22} i & \cdots & c a_{2 n} \\
\vdots & \vdots & & \vdots \\
c a_{n 1} & c a_{n 2} & \cdots & c y_{n n} i
\end{array}\right]
\end{aligned}
$$

diperoleh $c a_{i j} \in \mathbb{C}$ (sifat tertutup terhadap pergandaan pada bilangan kompleks). Ambil sebarang $c a_{i j} \in \mathbb{C}$ karena $a_{i j}=-\bar{a}_{j i} \quad \operatorname{maka} c a_{i j}=-\overline{c a}_{j i}$. Sesuai definisi 1 maka $c A \in \mathcal{S} H_{n}$.

\section{Sifat 5}

Jika $A \in \mathcal{S} H_{n}$ maka $\langle A \boldsymbol{x}, \boldsymbol{y}\rangle=\left\langle\boldsymbol{x}, A^{T} \boldsymbol{y}\right\rangle$ untuk setiap $\boldsymbol{x}, \boldsymbol{y} \in$ $\mathbb{C}^{n \times 1}$.

Bukti :

Ambil sebarang $A \in \mathcal{S} H_{n}$ dan $\boldsymbol{x}, \boldsymbol{y} \in \mathbb{C}^{\mathrm{n} \times 1}$

$$
A=\left[\begin{array}{cccc}
\alpha_{11} i & a_{12} & \cdots & a_{1 n} \\
a_{21} & \alpha_{22} i & \cdots & a_{2 n} \\
\vdots & \vdots & & \vdots \\
a_{n 1} & a_{n 2} & \cdots & \alpha_{n n} i
\end{array}\right]
$$

$$
\begin{aligned}
& \langle A \boldsymbol{x}, \boldsymbol{y}\rangle=\left\langle\left[\begin{array}{cccc}
\alpha_{11} i & a_{12} & \cdots & a_{1 n} \\
a_{21} & \alpha_{22} i & \cdots & a_{2 n} \\
\vdots & \vdots & & \vdots \\
a_{n 1} & a_{n 2} & \cdots & \alpha_{n n} i
\end{array}\right]\left[\begin{array}{c}
x_{1} \\
x_{2} \\
\vdots \\
x_{n}
\end{array}\right],\left[\begin{array}{c}
y_{1} \\
y_{2} \\
\vdots \\
y_{n}
\end{array}\right]\right\rangle \\
& =<\left[\begin{array}{c}
\alpha_{11} i x_{1}+a_{12} x_{2}+\cdots+a_{1 n} x_{n} \\
a_{21} x_{1}+\alpha_{22} i x_{2}+\cdots+a_{2 n} x_{n} \\
\vdots \\
a_{n 1} x_{1}+a_{n 2} x_{2}+\cdots+\alpha_{n n} i x_{n}
\end{array}\right],\left[\begin{array}{c}
y_{1} \\
y_{2} \\
\vdots \\
y_{n}
\end{array}\right]> \\
& =\left(\alpha_{11} i x_{1}+a_{12} x_{2}+\cdots+a_{1 n} x_{n}\right) y_{1} \\
& +\left(a_{21} x_{1}+\alpha_{22} i x_{2}+\cdots\right. \\
& \left.+a_{2 n} x_{n}\right) y_{2}+\left(a_{n 1} x_{1}\right. \\
& \left.+a_{n 2} x_{2}+\cdots+\alpha_{n n} i x_{n}\right) y_{n} \\
& =\alpha_{11} i x_{1} y_{1}+a_{12} x_{2} y_{1}+\cdots+a_{1 n} x_{n} y_{1} \\
& +a_{21} x_{1} y_{2}+\alpha_{22} i x_{2} y_{2}+\cdots \\
& +a_{2 n} x_{n} y_{2}+a_{n 1} x_{1} y_{n} \\
& +a_{n 2} x_{2} y_{n}+\cdots+\alpha_{n n} i x_{n} y_{n} \\
& =\alpha_{11} i x_{1} y_{1}+a_{21} x_{1} y_{2}+\cdots+a_{n 1} x_{1} y_{n} \\
& +a_{12} x_{2} y_{1}+\alpha_{22} i x_{2} y_{2}+\cdots \\
& +a_{n 2} x_{2} y_{n}+\cdots+a_{1 n} x_{n} y_{1} \\
& +a_{2 n} x_{n} y_{2}+\cdots+\alpha_{n n} i x_{n} y_{n} \\
& =x_{1}\left(\alpha_{11} i y_{1}+a_{21} y_{2}+\cdots+a_{n 1} y_{n}\right) \\
& +x_{2}\left(a_{12} y_{1}+\alpha_{22} i y_{2}+\cdots\right. \\
& \left.+a_{n 2} y_{n}\right)+\cdots+x_{n}\left(a_{1 n} y_{1}\right. \\
& \left.+a_{2 n} y_{2}+\cdots+\alpha_{n n} i y_{n}\right) \\
& =\left\langle\left[\begin{array}{c}
x_{1} \\
x_{2} \\
\vdots \\
x_{n}
\end{array}\right],\left[\begin{array}{cccc}
\alpha_{11} i & a_{21} & \cdots & a_{n 1} \\
a_{12} & \alpha_{22} i & \cdots & a_{n 2} \\
\vdots & \vdots & & \vdots \\
a_{1 n} & a_{2 n} & \cdots & \alpha_{n n} i
\end{array}\right]\left[\begin{array}{c}
y_{1} \\
y_{2} \\
\vdots \\
y_{n}
\end{array}\right]\right\rangle \\
& =\left\langle x, A^{T} y\right\rangle
\end{aligned}
$$

\section{KESIMPULAN}

Dari hasil pembahasan dan uraian pada bab-bab sebelumnya maka dapat diambil beberapa kesimpulan antara lain :

1. Sebuah matriks bujur sangkar merupakan matriks Skew Hermitian jika setiap elemenelemen penyusunnya merupakan bilangan kompleks beserta transpose konjugatnya dan matriks tersebut identik dengan negatif matriks transpose konjugatnya.

2. Beberapa sifat-sifat aljabar matriks yang berlaku pada matriks Skew Hermitian adalah sebagai berikut:

(i). Pengurangan suatu matriks kompleks dengan konjugatnya adalah matriks Skew Hermitian.

(ii). Suatu matriks kompleks merupakan jumlahan dari matriks Hermitian dan matriks Skew Hermitian.

(iii). Sebarang matriks Hermitian $A$ jika dan hanya jika $i A$ adalah Skew Hermitian.

(iv). Berlaku sifat tertutup terhadap penjumlahan 2 matriks Skew Hermitian dan terhadap pergandaan skalar. 
(v). Jika berlaku sifat komutatif pada pergandaan matriks $(A B=B A)$ maka $A B$ adalah matriks Skew Hermitian.

(vi). Himpunan matriks Skew Hermitian merupakan ruang vektor $\mathbb{R}$.

(vii).Untuk sebarang matriks Skew Hermitian berlaku $\langle A x, y\rangle=\left\langle x, A^{T} y\right\rangle$ untuk semua $x, y \in C^{n \times 1}$.

\section{DAFTAR PUSTAKA}

Hadley, G, 1983, Aljabar Linear, Edisi Revisi, Penerbit Erlangga, Jakarta.

Hogben, Leslie, 2007, Handbook of Linear Algebra.

Dalam. Barret, Wayne, (1973), Hermitian and Positive Definite Matrices, Taylor \& Francis, Group, USA: 130-131.

Michael,E,O’Sullivan,(2013), Lecture Notes for Math 623 Matrix Analysis.

Paliouras, John D, 1975, Peubah Kompleks untuk Ilmuwan dan Insinyur, Penerbit Erlangga, Jakarta.

Spiegel, Murray R, Teori dan Soal-soal Peubah Kompleks, Seri Buku Schaum, Penerbit Erlangga, Jakarta.

Wolfram, 1999, Hermitian Matrix - from Wolfram MathWorld 